\title{
Phase II study of S-1, a novel oral fluoropyrimidine, and biweekly administration of docetaxel for previously treated advanced non-small-cell lung cancer
}

\author{
Yasunari Oki • Takashi Hirose $\cdot$ Toshimitsu Yamaoka • Sojiro Kusumoto • \\ Takao Shirai · Tomohide Sugiyama · Kentaro Okuda • Masanao Nakashima • \\ Yasunori Murata $\cdot$ Tohru Ohmori $\cdot$ Mitsuru Adachi
}

Received: 7 April 2010 / Accepted: 2 June 2010 / Published online: 17 June 2010

(C) The Author(s) 2010. This article is published with open access at Springerlink.com

\begin{abstract}
Purpose We examined the safety and efficacy of the combination of S-1 and biweekly docetaxel in patients with previously treated advanced non-small-cell lung cancer (NSCLC).

Methods Patients with previously treated advanced NSCLC were eligible if they had a performance status of 2 or less, were 80 years or younger, and had adequate organ function. Forty-nine patients (38 men and 11 women; median age, 66 years; range 43-79 years) were enrolled. Patients were treated with the combination of $80 \mathrm{mg} / \mathrm{m}^{2}$ per day of S- 1 for 14 consecutive days and $35 \mathrm{mg} / \mathrm{m}^{2}$ of docetaxel on days 1 and 15 every 4 weeks.

Results The overall response rate was $16.3 \%$ (95\% confidence interval, 7.6-30.5\%). The disease-control rate was $49.0 \%$ (95\% confidence interval, $34.4-63.7 \%$ ). The median survival time after this treatment was 9 months (range 1-22 months). The median progression-free survival time was 3 months (range 1-11 months). Response rates and survival times did not differ significantly according to the histological type. Grade 3-5 toxicities included neutropenia in $51.0 \%$ of patients, thrombocytopenia in $2.0 \%$, anemia in $20.4 \%$, infection in $24.5 \%$, anorexia in $12.2 \%$, diarrhea in $14.3 \%$, nausea in $6.1 \%$, and dehydration in $4.2 \%$. There
\end{abstract}

Y. Oki · T. Hirose $(\bowtie) \cdot$ T. Yamaoka · S. Kusumoto · T. Shirai · T. Sugiyama $\cdot$ K. Okuda $\cdot$ M. Nakashima $\cdot$ Y. Murata $\cdot$ M. Adachi Division of Respiratory Medicine and Allergology,

Department of Internal Medicine,

Showa University School of Medicine,

1-5-8 Hatanodai, Shinagawa, Tokyo 142-8666, Japan

e-mail: thirose-shw@umin.ac.jp

T. Ohmori

Institute of Molecular Oncology,

Showa University School of Medicine,

1-5-8 Hatanodai, Shinagawa, Tokyo 142-8666, Japan was 1 treatment-related death due to severe anorexia, stomatitis, diarrhea, and, as consequence, dehydration.

Conclusions The combination of S-1 and biweekly docetaxel is an acceptable therapeutic option in patients with previously treated advanced NSCLC regardless of the histological type.

Keywords S-1 - Docetaxel - Second-line chemotherapy · Non-small-cell lung cancer

\section{Introduction}

Although platinum-based chemotherapy improves survival over best supportive care for patients with chemotherapynaïve advanced non-small-cell lung cancer (NSCLC) [1], almost all patients relapse after receiving first-line chemotherapy. The prognosis of patients who have relapsed or have refractory NSCLC and do not receive additional therapy is extremely poor: median survival time (MST) is reported to be only $4-5$ months $[2,3]$. On the other hand, many patients maintain a good performance status (PS) and have adequate organ function. Thus, second-line chemotherapy is an important treatment for patients with previously treated advanced NSCLC.

Docetaxel promotes microtubule assembly and inhibits depolymerization to free tubulin, which results in the arrest of the cell cycle in the M phase [4]. In 2 phase III trials in patients with NSCLC previously treated with a platinumbased regimen, docetaxel confers greater survival and clinical benefits and improves quality of life to a greater degree than does best supportive care or monotherapy with vinorelbine or ifosfamide $[2,5]$. As a result of these randomized trials, docetaxel has become one of standard treatments for NSCLC refractory to platinum-based agents. However, 
these trials yielded unsatisfactory results, with response rates of approximately 7\%, time to progression of 10.6 and 8.5 weeks, MSTs of 7.5 and 5.7 months, and 1-year survival rates of 37 and $32 \%$, respectively [2, 5].

S-1 was developed to enhance the efficacy and reduce the toxicity of 5-fluorouracil (5-FU). S-1 is an oral fluoropyrimidine agent that consists of tegafur, which is a prodrug of 5-FU, and two biochemical modulators, 5-chloro-2, 4-dihydroxypyridine (CDHP), and potassium oxonate in a molar ratio of 1:0.4:1 [6]. CDHP competitively inhibits dihydropyrimidine dehydrogenase, a key enzyme for 5-FU degradation, and enhances the anticancer activity of 5-FU. Potassium oxonate inhibits the phosphorylation of 5-FU in the gut by inhibiting the enzymatic pyrimidine phosphoribosyl transferase pathway and reduces the gastrointestinal toxicity of active 5-FU without interfering with its antitumor activity [7]. S-1 monotherapy has achieved an overall response rate of $22 \%$ and a MST of 11 months with few severe gastrointestinal or hematologic adverse events in patients with advanced NSCLC [8].

In preclinical studies, docetaxel has shown no crossresistance in 5-FU-resistant human tumor cell lines [9]. Additionally, the combination of S-1 and docetaxel has been reported to have synergistic effects in vitro [10]. Moreover, in xenograft models of gastric and breast cancer the combination of S-1 and docetaxel has shown synergistic antitumor activity compared with monotherapy with either drug [11, 12]. Furthermore, S-1 and docetaxel have different mechanisms of action and safety profiles and show no pharmacokinetic interaction [11]. Therefore, the doublet combination chemotherapy of S-1 and docetaxel would be more effective than docetaxel monotherapy.

Several phase II studies have found that the combination of docetaxel and S-1 is effective in patients with lung or gastric cancer [13-16]. A phase I trial of the combination of biweekly docetaxel and S-1 for recurrent advanced gastric cancer has recommended dose levels for phase II studies of $35 \mathrm{mg} / \mathrm{m}^{2}$ of docetaxel on days 1 and 15 in combination with $80 \mathrm{mg} / \mathrm{m}^{2}$ per day of S-1 for 14 consecutive days every 4 weeks [17]. However, no trials have evaluated the combination of S-1 and biweekly docetaxel in patients with previously treated advanced NSCLC. Therefore, we performed the present phase II study to assess the antitumor activity and toxicity of S-1 and biweekly docetaxel, with dose levels based on the above phase I study, in patients with previously treated advanced NSCLC. The primary end point of present phase II study was to assess the response rates, and the secondary endpoints were to assess the safety, progression-free survival, and overall survival of S-1 and biweekly docetaxel in patients with previously treated advanced NSCLC.

\section{Patients and methods}

\section{Eligibility criteria}

Patients with NSCLC who had previously received cytotoxic chemotherapy were enrolled. The criteria for study entry were as follows: (1) histologically or cytologically confirmed NSCLC; (2) stage III or IV disease; (3) age 80 or less; (4) Eastern Cooperative Oncology Group PS of 2 or less; (5) measurable or assessable lesions; (6) life expectancy of at least 8 weeks; (7) adequate bone marrow function (white blood cell count of 4,000-12,000/ $\mu 1$, neutrophil count of $2,000 / \mu 1$ or more, platelet count of $100,000 / \mu 1$ or more, and hemoglobin level of $9 \mathrm{~g} / \mathrm{dl}$ or more), hepatic function (total serum bilirubin level less than the upper limit of the normal range, levels of aspartate aminotransferase and alanine aminotransferase less than or equal to twice the upper limits of the normal ranges), and renal function (serum creatinine level less than $1.5 \mathrm{mg} / \mathrm{dl}$ and creatinine clearance rate of $50 \mathrm{ml} / \mathrm{min}$ or more). Patients were excluded if they had pulmonary fibrosis or interstitial pneumonia, severe heart disease, active infection, severe drug allergy, other serious underlying medical conditions, symptomatic brain metastasis, or an active second malignancy. The study protocol was approved by the ethics committee of Showa University School of Medicine, and all patients provided written informed consent.

Treatment schedule

S-1 was given orally twice daily after a meal for 14 consecutive days, followed by 14 days of rest. The dose of S-1 ( $80 \mathrm{mg} / \mathrm{m}^{2}$ per day) was simplified according to body surface area (BSA) as follows: $80 \mathrm{mg} /$ day for patients with a BSA of less than $1.25 \mathrm{~m}^{2}, 100 \mathrm{mg} /$ day for those with a BSA of $1.25-1.5 \mathrm{~m}^{2}$, and $120 \mathrm{mg}$ /day for those with a BSA greater than $1.5 \mathrm{~m}^{2}$. Docetaxel $\left(35 \mathrm{mg} / \mathrm{m}^{2}\right)$ was diluted in $500 \mathrm{ml}$ of normal saline and given as an intravenous drip infusion over $60 \mathrm{~min}$ on days 1 and 15 . This regimen was repeated every 4 weeks for a maximum of six courses. Chemotherapy was discontinued for grade 3 or higher nonhematologic toxicity, except for nausea/vomiting, anorexia, stomatitis, diarrhea, constipation, alopecia, or fatigue at any time, or if the treatment outcome was progressive disease at any time. If 2 or more weeks had passed after the scheduled start of the next course until these criteria were satisfied, the patient left the study at that time but was still included in the analysis. If there was stable disease after two courses, subsequent therapy was left to the discretion of the physician in charge of the patient. Palliative radiotherapy was permitted to control persistent pain associated with bone metastasis.

The next course of treatment was started when the neutrophil count returned to $1,500 / \mu 1$, the platelet count returned 
to $75,000 / \mu 1$, and the nonhematologic toxicity recovered to grade 0 or 1 . Administration of S-1 was stopped if the neutrophil count was less than $500 / \mu$ l or if the platelet count was less than $20,000 / \mu 1$ and was restarted when neutrophil count returned to $1,500 / \mu \mathrm{l}$ and the platelet count returned to $75,000 / \mu \mathrm{l}$. Administration of docetaxel was delayed 1 week if the neutrophil count was less than $1,000 / \mu l$ or if the platelet count was less than $75,000 / \mu 1$, and the dose of docetaxel was omitted if the neutrophil count or the platelet count had not recovered by 1 week later. If administration of docetaxel was delayed 1 week, administration of S-1 at the next course was also delayed 1 week. Additionally, if administration of S-1 was stopped, and delayed the last planned dose of S-1, administration of docetaxel at the next course was also delayed according to the day of the last administration of S-1. If grade 2 nonhematologic toxicities, except for nausea, anorexia, fatigue, alopecia, or hepatic dysfunction, occurred, administration of S-1 was continued but doses were reduced by $20 \mathrm{mg} /$ day. For the next course, docetaxel doses were reduced by $5 \mathrm{mg} / \mathrm{m}^{2}$, and $\mathrm{S}-1$ doses were reduced by $20 \mathrm{mg} /$ day if the patient had grade 4 neutropenia lasting 3 days or longer, grade 4 thrombocytopenia, or grade 3 or 4 neutropenia associated with a fever higher than $38^{\circ} \mathrm{C}$. The $\mathrm{S}-1$ doses were reduced by $20 \mathrm{mg} /$ day if S-1 administration was stopped for longer than 8 days. Dexamethasone and the antiemetic medication ondansetron were given prophylactically to all patients before docetaxel administration. To prevent and counteract neutropenia, we routinely used granulocyte colony-stimulating factor until neutrophil counts had returned to the normal range in patients with grade 4 leukopenia or neutropenia, according to the guideline of the Japanese Ministry of Health, Labour and Welfare.

\section{Evaluation}

Evaluation before treatment included a baseline history and physical examination, complete blood count with differential, routine chemistry profiles, chest radiography, computed tomography of the chest and abdomen, magnetic resonance or computed tomography of the brain, and a radionucleotide bone scan. Complete blood counts with differential and routine chemistry profiles were determined at least once a week during chemotherapy. Chest radiography was performed once per week during chemotherapy, and electrocardiography was performed before and after chemotherapy.

Tumors were measured after the second, fourth, and sixth courses. Tumor response was classified according to Response Evaluation Criteria in Solid Tumors (RECIST Guidelines) [18]. Toxicities were assessed and graded according to the National Cancer Institute Common Terminology Criteria for Adverse Events, version 3.0. All patients who had received at least 2 courses of chemotherapy were assessable for response, and all patients who had received at least 1 course of chemotherapy were assessable for toxicity and survival.

\section{Statistical methods}

The trial was designed as a phase II study, with response rate as the main endpoint. We chose a $30 \%$ response rate as a desirable target level and a $10 \%$ response rate as undesirable. The study design had a power of greater than $90 \%$ to detect a response with an error of less than $5 \%$. Therefore, we required 42 assessable patients according to Simon's minimax design.

Progression-free survival (PFS) time was defined as the period from the start of this treatment to the identifiable time for first progression or death from any cause. Survival time was measured from the start of the present treatment until death or last follow-up. Survival curves were constructed with the Kaplan-Meier method and were analyzed with the log-rank test. The chi-square test was used to determine the significance of differences in response rates between squamous cell carcinoma and other types of cancer and between patients who received and did not receive paclitaxel pretreatment and of differences in toxicity between patients of different age. Differences with a $p$ value $<0.05$ were considered statistically significant.

\section{Results}

Patients characteristics

From October 2006 through March 2009, 49 patients were enrolled (Table 1). Of these 49 patients, $16(32.7 \%)$ were 70 years or older and $7(14.3 \%)$ had a PS of 2 . The median time from the end of the last previous chemotherapy to the present treatment was 5 months (range 0.5-31 months). Toxicity and survival could be assessed in all 49 patients, and response could be assessed in 45 patients. Four patients could not be evaluated for response because they had not received two courses of chemotherapy owing to their general condition having rapidly deteriorated after receiving chemotherapy (three patients) and to patient refusal (one patient). These patients were considered not evaluable, i.e., they were included in the denominator but not in the numerator in calculations of the response rate and the disease-control rate.

\section{Treatments before or after the present treatment}

All patients had received a platinum-containing regimen as first-line chemotherapy: carboplatin and vinorelbine (13 patients), cisplatin and vinorelbine (11 patients), nedaplatin 
Table 1 Patients characteristics

\begin{tabular}{ll}
\hline Total number of patients & 49 \\
Sex (M/F) & $38 / 11$ \\
Age, years (range) & $66(43-79)$ \\
Performance status at relapse (0/1/2) & $4 / 38 / 7$ \\
Stage at relapse (IIIA/IIIB/IV) & $3 / 7 / 39$ \\
Histologic type & \\
Adenocarcinoma & 34 \\
Squamous & 11 \\
Large & 2 \\
Others & 2 \\
Number of prior regimens of chemotherapy (1/2) & $42 / 7$ \\
Thoracic radiation therapy (yes/no) & $22 / 27$ \\
Response to previous chemotherapy & \\
Complete response & 5 \\
Partial response & 23 \\
Stable disease & 7 \\
Progressive disease & 13 \\
Not evaluable & 1 \\
\hline
\end{tabular}

and paclitaxel (10 patients), nedaplatin and gemcitabine (7 patients), carboplatin and gemcitabine (5 patients), and carboplatin and paclitaxel (3 patients). Thus, 10 patients had previously been treated with paclitaxel. All 7 patients who underwent this treatment as third-line chemotherapy had previously received gefitinib or erlotinib, which are epidermal growth factor receptor (EGFR) tyrosine kinase inhibitors, as second-line therapy. After this treatment, 14 (29\%) patients received an EGFR tyrosine kinase inhibitor alone, $10(35 \%)$ patients received cytotoxic chemotherapy alone, and seven patients (20\%) received both an EGFR tyrosine kinase inhibitor and cytotoxic chemotherapy.

Treatment response and survival

Of the 49 patients, $1(2.0 \%)$ achieved a complete response, $7(14.3 \%)$ achieved a partial response, $16(32.6 \%)$ had stable disease, $21(42.9 \%)$ had progressive disease, and 4 $(8.2 \%)$ were not evaluable, for an overall response rate of $16.3 \%$ [95\% confidence interval (CI), 7.3-29.7\%]. The disease-control rate was $49.0 \%$ (95\% CI, 34.4-63.7\%). Response rates were $14.7 \%$ for adenocarcinoma, $18.2 \%$ for squamous cell carcinoma, and $25.0 \%$ for other types and did not differ significantly according to the histological type $(p=0.95)$. Response rate did not differ significantly between patients who had received paclitaxel pretreatment $(15.4 \%)$ and patients who had not $(16.7 \%, p=0.62)$.

Survival analysis was performed when the median follow-up time of all evaluable patients was 9 months. At the time of analysis, nine patients $(18.4 \%)$ were alive and none had been lost to follow-up. The MST from the start of this

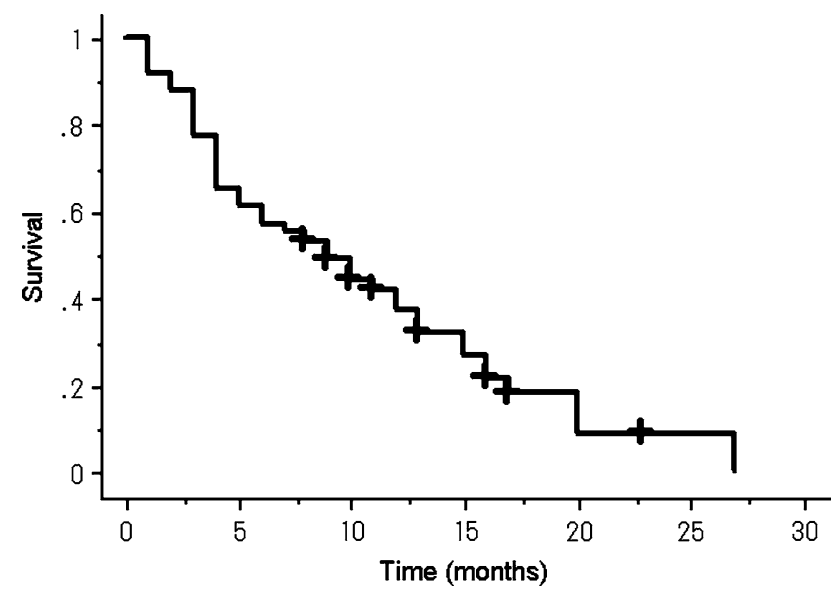

Fig. 1 The figure shows overall survival from this treatment estimated with the Kaplan-Meier method. The median survival time was 9 months (range 1-22 months)

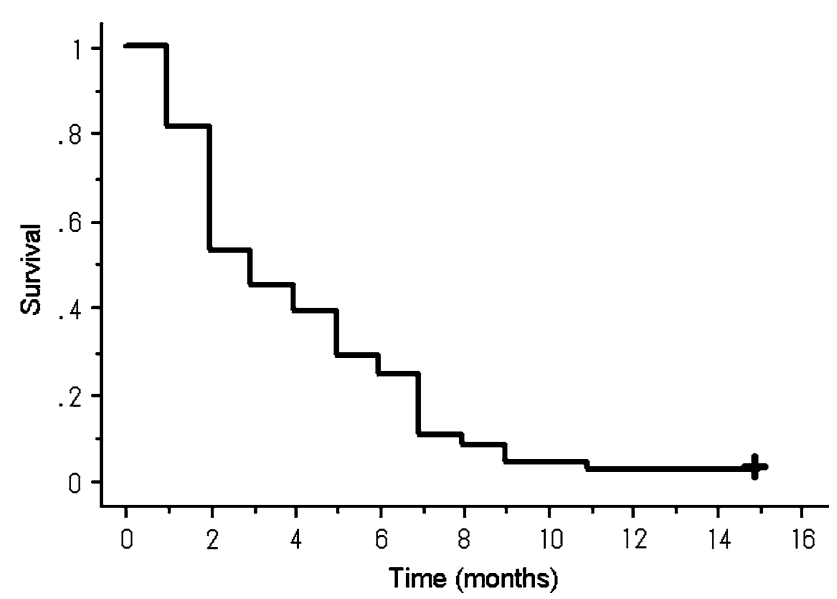

Fig. 2 The figure shows progression-free survival time from this treatment estimated with the Kaplan-Meier method. The median progression-free survival time was 3 months (range 1-11 months)

regimen was 9 months (range 1-22 months; Fig. 1). The 1 -year survival rate from the start of this regimen was $42 \%$. The MST did not differ significantly according to the histological type $(p=0.28)$. The MST did not differ significantly between patients who had received paclitaxel pretreatment and patients who had not $(p=0.35)$. The median PFS time was 3 months (range 1-15 months; Fig. 2).

\section{Toxicity}

Table 2 lists the worst-grade toxicities developing during treatment. Grade 3-4 hematologic toxicities included neutropenia in $51.0 \%$ of patients, thrombocytopenia in $2.0 \%$, and anemia in $20.4 \%$. Granulocyte colony-stimulating factor was given during $20.7 \%$ of courses (25 of 121 courses; median duration of administration, 3 days; range 1-8 days). One patient received platelet transfusions, and 4 patients 
Table 2 Toxicity

\begin{tabular}{|c|c|c|c|c|c|c|}
\hline \multirow[b]{2}{*}{ Toxicity } & \multicolumn{6}{|c|}{$\begin{array}{l}\text { National Cancer Institute-Common } \\
\text { Terminology Criteria grade }\end{array}$} \\
\hline & 1 & 2 & 3 & 4 & 5 & $3-5(\%)$ \\
\hline Leukopenia & 1 & 13 & 14 & 4 & 0 & 36.7 \\
\hline Neutropenia & 5 & 6 & 9 & 16 & 0 & 51.0 \\
\hline Thrombocytopenia & 14 & 4 & 0 & 1 & 0 & 2.0 \\
\hline Anemia & 19 & 20 & 9 & 1 & 0 & 20.4 \\
\hline Nausea & 11 & 8 & 3 & 0 & 0 & 6.1 \\
\hline Vomiting & 11 & 3 & 0 & 0 & 0 & 0 \\
\hline Anorexia & 15 & 10 & 4 & 1 & 1 & 12.2 \\
\hline Stomatitis & 8 & 8 & 3 & 0 & 0 & 6.1 \\
\hline Diarrhea & 13 & 10 & 5 & 1 & 1 & 14.3 \\
\hline Dehydration & 0 & 9 & 0 & 1 & 1 & 4.1 \\
\hline Infection & 0 & 3 & 12 & 0 & 0 & 24.5 \\
\hline Elevation of serum creatinine & 4 & 1 & 0 & 0 & 0 & 0 \\
\hline Elevation of aminotransferases & 13 & 1 & 2 & 0 & 0 & 4.1 \\
\hline Hypokalemia & 19 & 1 & 5 & 0 & 0 & 10.2 \\
\hline Hyponatremia & 38 & 0 & 4 & 0 & 0 & 8.2 \\
\hline Fatigue or asthenia & 8 & 7 & 3 & 0 & 0 & 6.1 \\
\hline Nail/skin toxicity & 4 & 9 & 2 & 0 & 0 & 4.1 \\
\hline
\end{tabular}

received erythrocyte transfusions. No patients had severe complications related to myelosuppression.

Although the most frequent nonhematologic toxicities were electrocyte abnormalities, all electrocyte abnormalities resolved soon without medication or hydration. Grade 3-5 nonhematologic toxicities included infection in $24.5 \%$ of patients, anorexia in $12.2 \%$, diarrhea in $14.3 \%$, nausea in $6.1 \%$, and dehydration in $4.2 \%$. Although 1 patient had grade 3 pulmonary toxicity due to Pneumocystis carinii pneumonia during the second course, this patient recovered after receiving trimethoprim-sulfamethoxazole, corticosteroids, and supplemental oxygen. There was no drug-induced pneumonitis. On the other hand, there was 1 treatmentrelated death. This patient was a 71-year-old man with a PS of 1 and normal renal function, who died during the second course, because of stomatitis and diarrhea, and, as consequence, dehydration developed after the completion of administration of S-1 for 14 consecutive days and administration of docetaxel on days 1 and 15 .

Table 3 shows the frequency of toxicity according to age. Stomatitis, dehydration, and infection were significantly more frequent in patients 70 years or older than in patients younger than 70 years.

Dose intensity

A total of 121 courses of chemotherapy were given. The median number of courses given per patient was 2 (range $1-6)$. Of the 49 patients, 4 (8.2\%) discontinued this treatment:
Table 3 Grade 3-5 toxicity according to age

\begin{tabular}{llll}
\hline & $\begin{array}{l}70 \text { years or } \\
\text { older } \\
(n=16)\end{array}$ & $\begin{array}{l}\text { Younger } \\
\text { than 70 years } \\
(n=33)\end{array}$ & $p$ \\
\hline Leukopenia & $5(31.3 \%)$ & $13(39.4 \%)$ & 0.579 \\
Neutropenia & $11(68.8 \%)$ & $14(42.4 \%)$ & 0.084 \\
Anemia & $4(25 \%)$ & $6(18.9 \%)$ & 0.583 \\
Thrombocytopenia & $1(6.3 \%)$ & $0(0 \%)$ & 0.147 \\
Nausea & $2(12.5 \%)$ & $1(3.0 \%)$ & 0.195 \\
Stomatitis & $3(18.8 \%)$ & $0(0 \%)$ & 0.01 \\
Anorexia & $4(25.0 \%)$ & $2(6.1 \%)$ & 0.058 \\
Diarrhea & $4(25.0 \%)$ & $3(9.1 \%)$ & 0.136 \\
Dehydration & $2(12.5 \%)$ & $0(0 \%)$ & 0.038 \\
Infection & $8(50.0 \%)$ & $4(12.1 \%)$ & 0.004 \\
\hline
\end{tabular}

the causes were grade 3 infection in two patients, grade 3 hepatic dysfunction in one patient, and patient refusal due to grade 2 nausea in one patient. In addition, doses of docetaxel on day 15 were skipped in four patients because of neutropenia, infection, or disease progression. Doses of both docetaxel and S-1 administration were reduced in two patients because of grade 4 neutropenia lasting 3 days or neutropenic fever. Additionally, doses of S-1 were reduced in 12 patients mainly because of diarrhea, vomiting, stomatitis, or infection. The next course of chemotherapy was delayed after $12(9.9 \%)$ of the 121 courses, mainly because of neutropenia or diarrhea. In the 49 patients, dose intensities of docetaxel decreased in 19 patients (38.8\%), and dose intensities of S-1 decreased in 23 patients $(46.9 \%)$. The mean percentage of individual actually delivered doses per planned doses was $94.1 \%$ for docetaxel and $92.7 \%$ for S-1.

\section{Discussion}

Several randomized phase III trials in patients with previously treated advanced NSCLC have demonstrated that docetaxel or erlotinib prolongs survival and decreases symptoms when compared with placebo [2, 3]. Moreover, in patients with previously treated NSCLC pemetrexed or gefitinib achieves efficacy outcomes clinically equivalent to those of docetaxel $[19,20]$. Thus, docetaxel, pemetrexed, gefitinib, or erlotinib is recommended as a second-line therapy for advanced NSCLC [21]. However, the efficacy remains poor: reported response rates in unselective previously treated NSCLC are 6.7 to $10.8 \%$ with docetaxel, $9.1 \%$ with pemetrexed, $9.1 \%$ with gefitinib, and $8.9 \%$ with erlotinib, and 1-year survival rates are 25 to $37 \%$ [2, 3, 5, 19, 20]. Therefore, further research should focus on the development of regimens that are more effective against previously treated advanced NSCLC. 
In a recent phase III study in patients with previously treated NSCLC, weekly docetaxel at a dose of $35 \mathrm{mg} / \mathrm{m}^{2}$ was similarly efficacious as and better tolerated-specifically, incidences of grade 3-4 neutropenia and anemia were lower-than a 3-weekly docetaxel at a dose of $75 \mathrm{mg} / \mathrm{m}^{2}$ [22]. On the other hand, in a phase II trial of biweekly docetaxel at a dose of $50 \mathrm{mg} / \mathrm{m}^{2}$ as second-line treatment in patients with NSCLC, grade 3 or 4 neutropenia developed in only $16 \%$ of patients [23]. Therefore, to reduce toxicity we chose biweekly administration of docetaxel.

In the present study, the overall response rate in previously treated patients with a PS of 0-2 was $16.3 \%$ with a MST of 9 months, a 1-year survival rate of $42 \%$, and a median PFS time of 3 months. Two previous studies have reported that docetaxel at a dose of $40 \mathrm{mg} / \mathrm{m}^{2}$ on day 1 and S-1 at a dose of $80 \mathrm{mg} / \mathrm{m}^{2}$ per day on days $1-14$ every 3 weeks in patients with previously treated advanced NSCLC and a PS of 0 or 1 achieved overall response rates of 24.1 and $18.4 \%$ with MSTs of 11.8 months and 16.1 months, 1-year survival rates of 42 and $60 \%$, and median PFS times of 3.9 months and 4.4 months, respectively $[13,14]$. Our response and survival rates were slightly lower than those of studies reported by Atagi et al. and Yanagihara et al. [13, 14]. The reason might be that neither previous report included patients with a PS of 2, whereas our study included seven patients (14.3\%) with a PS of 2. Some previous trials have suggested that patients with a PS of 2 have significantly lower rates of response and survival and higher rates of toxicity than do patients with a PS of 0 or $1[24,25]$. On the other hand, our results compare favorably with those of most published trials of the second-line treatment of NSCLC, although no conclusions can be drawn from a direct comparison of the present results with previous results. In previous randomized phase III studies for previously treated NSCLC, overall response rates have ranged from 6.7 to $20 \%$, MSTs have ranged from 5.4 to 10.2 months, 1-year survival rates have ranged from 19 to $45.6 \%$, and median PFS times have ranged from 2.1-5.6 months [2, 5, 19, 22, 26-28].

We found that prior paclitaxel treatment status did not significantly affect the response rate or the survival time. Our results were consistent with those of previous phase III trials of docetaxel [5, 22]. Therefore, this regimen including docetaxel should be effective in patients who have previously been treated with paclitaxel.

Pemetrexed has been reported to be effective against adenocarcinoma but not against squamous cell carcinoma of the lung [29]. In addition, although patients with NSCLC harboring an activating mutation in the EGFR gene respond well to EGFR tyrosine kinase inhibitors, activating mutations of the EGFR gene are present mostly in patients with adenocarcinoma [30, 31]. Thus, options for second-line treatment for patients with squamous cell carcinoma are more limited than for patients with adenocarcinoma. In our study, response rates and survival times did not differ significantly with the histological type, although only small numbers of patients were evaluated. Therefore, we believe this regimen would be effective regardless of the histological type.

In the present study, grade 3-4 hematologic toxicities, including neutropenia, developed in $51.0 \%$ of patients, thrombocytopenia developed in $2.0 \%$, and anemia developed in $20.4 \%$. In addition, grade 3-5 nonhematologic toxicities included infection in $24.5 \%$ of patients, anorexia in $12.2 \%$, diarrhea in $14.3 \%$, nausea in $6.1 \%$, and dehydration in $4.2 \%$. There was one treatment-related death due to severe anorexia, stomatitis and diarrhea, and, as consequence, dehydration. This patient was a 71-year-old man. Grade 3-5 stomatitis, dehydration, and infection were significantly more frequent in patients 70 years or older than in patients younger than 70 years. Toxicities observed in this study were generally tolerable. However, the incidences of grade 3-4 neutropenia, anemia, and grade 3-5 diarrhea in our study were higher than those of studies reported by Atagi et al. and Yanagihara et al. or those in recently published trials of single-agent docetaxel or pemetrexed, whereas the incidences of the above toxicities in our study were similar to or higher than those of previous trials of the combination of docetaxel and gemcitabine or irinotecan $[2,5,13,14,19,22,26-28]$. Zang et al. [32] have reported that the dosages of the combination of docetaxel and S-1 should be modified according to the patient's age, because grade 4 neutropenia or neutropenic fever occur more frequently in patients older than 60 years. In contrast, in a subset analysis of phase III trials comparing single-agent pemetrexed with docetaxel in patients with previously treated NSCLC, there was no significant difference in toxicity between patients 70 years or older and patients younger than 70 years [33]. Thus, single-agent chemotherapy might be more appropriate than doublet chemotherapy in previously treated patients 70 years or older.

In conclusion, to our knowledge, the present phase II study is the first to examine the combination of S-1 and biweekly docetaxel for previously treated advanced NSCLC. This treatment is safe and effective in patients with previously treated advanced NSCLC and is, therefore, an acceptable option in this setting regardless of the histological type. A phase III study comparing docetaxel alone to docetaxel plus S-1 in patients with previously treated advanced NSCLC has begun enrollment in Japan to determine whether adding S-1 to docetaxel is clinically beneficial.

Conflict of interest statement None. 
Open Access This article is distributed under the terms of the Creative Commons Attribution Noncommercial License which permits any noncommercial use, distribution, and reproduction in any medium, provided the original author(s) and source are credited.

\section{References}

1. NSCLC Meta-Analyses Collaborative Group (2008) Chemotherapy in addition to supportive care improves survival in advanced non-small-cell lung cancer: a systemic review and meta-analysis of individual patient data from 16 randomized control trials. J Clin Oncol 26:4617-4625

2. Shepherd FA, Dancey J, Ramlau R et al (2000) Prospective randomized trial of docetaxel versus best supportive care in patients with non-small-cell lung cancer previously treated with platinumbased chemotherapy. J Clin Oncol 18:2095-2103

3. Shepherd FA, Pereira JR, Ciuleanu T et al (2005) Erlotinib in previously treated non-small cell lung cancer. N Engl J Med 353:123132

4. Ringel I, Horwitz SB (1991) Studies with RP 56976 (Taxotere): a semisynthetic analogue of Taxol. J Natl Cancer Inst 83:288-291

5. Fossella FV, Devore R, Kerr RN et al (2000) Randomized phase III trial of docetaxel versus vinorelbine or ifosfamide in patients with advanced non-small cell lung cancer previously treated with platinum-containing chemotherapy regimens. J Clin Oncol 18:2354-2362

6. Shirasaka T, Nakano K, Takechi T et al (1996) Antitumor activity of $1 \mathrm{M}$ tegarur- $0.4 \mathrm{M}$ 5-chloro-2, 4-dihydroxypyridine-1 $\mathrm{M}$ potassium oxonate (S-1) against human colon carcinoma orthotopically implanted into nude rats. Cancer Res 56:2602-2606

7. Shirasaka T, Shimamoto Y, Fukushima M (1993) Inhibition by oxonic acid of gastrointestinal toxicity of 5-fluorouracil without loss of its antitumor activity in rats. Cancer Res 53:4004-4009

8. Kawahara M, Furuse K, Segawa Y et al (2001) Phase II study of $\mathrm{S}-1$, a novel oral fluorouracil, in advanced non-small-cell lung cancer. Br J Cancer 85:939-943

9. Hill BT, Whelan RDH, Shellard SA et al (1994) Differential cytotoxic effects of docetaxel in a range of mammalian tumor cell lines and certain drug resistant sublines in vitro. Invest New Drugs 12:169-182

10. Wada Y, Yoshida K, Suzuki T et al (2006) Synergistic effects of docetaxel and S-1 by modulating the expression of metabolic enzymes of 5-fluorouracil in human gastric cancer cell lines. Int J Cancer 119:783-791

11. Takahashi I, Emi Y, Kakeji Y et al (2005) Increased antitumor activity in combined treatment TS-1 and docetaxel. Oncology 68:130-137

12. Suto A, Kubota T, Fukushima M et al (2006) Antitumor effect of combination of S-1 and docetaxel on the human breast cancer xenograft transplanted into SCID mice. Oncol Rep 15:1517-1522

13. Atagi S, Kawahara M, Kusunoki Y et al (2008) Phase I/II study of docetaxel and S-1 in patients with previously treated non-small cell lung cancer. J Thorac Oncol 3:1012-1017

14. Yanagihara K, Yoshimura K, Niimi M et al (2010) Phase II study of S-1 and docetaxel for previously treated patients with locally advanced or metastatic non-small cell lung cancer. Cancer Chemother Pharmacol (Epub ahead of print)

15. Yoshida K, Ninomiya M, Takakura N et al (2006) Phase II study of docetaxel and S-1 combination therapy for advanced or recurrent gastric cancer. Clin Cancer Res 12:3402-3407

16. Yamaguchi K, Shimamura T, Hyodo I et al (2006) Phase I/II study of docetaxel and S-1 in patients with advanced gastric cancer. Br J Cancer 94:1803-1808
17. Takahashi I, Emi Y, Kakeji Y et al (2006) Phase I study of S-1 and biweekly docetaxel combination chemotherapy for advanced and recurrent gastric cancer. Oncol Rep 15:849-854

18. Therasse P, Arbuck SG, Eisenhauer EA et al (2000) New guidelines to evaluate the response to treatment in solid tumors (RECIST Guidelines). J Natl Cancer Inst 92:205-216

19. Hanna N, Shepherd FA, Fossela FV et al (2004) Randomized phase III trial of pemetrexed versus docetaxel in patients with nonsmall-cell lung cancer previously treated with chemotherapy. J Clin Oncol 22:1589-1597

20. Kim ES, Hirsh V, Mok T et al (2008) Gefitinib versus docetaxel in previously treated non-small-cell lung cancer (INTEREST): a randomized phase III trial. Lancet 372:1809-1818

21. Azzoli CG, Baker S Jr, Temin S et al (2009) American Society of Clinical Oncology Clinical Practice Guideline Update on chemotherapy for stage IV non-small-cell lung cancer. J Clin Oncol 27:6251-6266

22. Shuette W, Nagel S, Blankenburg T et al (2005) Phase III study of second-line chemotherapy for advanced non-small-cell lung cancer with weekly compared with 3-weekly docetaxel. J Clin Oncol 23:8389-8395

23. Vazquez S, Grande C, Amenedo M et al (2004) Biweekly docetaxel as second-line chemotherapy of patients with advanced non-small cell lung cancer: a phase II study of the Galician Lung Cancer Group (GGCP 006-00). Anti-Cancer Drugs 15:489-494

24. Sweeney CJ, Zhu J, Sandler AB et al (2001) Outcome of patients with a performance status of 2 in Eastern Cooperative Oncology Group Study E1594. Cancer 92:2639-2647

25. Helbekkmo N, Aasebo U, Sundstrom SH et al (2008) Treatment outcome in performance status 2 advanced NSCLC patients administered platinum-based combination chemotherapy. Lung Cancer 62:253-260

26. Pectasides D, Pectasides M, Farmakis D et al (2005) Comparison of docetaxel and docetaxel-irinotecan combination as second-line chemotherapy in advanced non-small-cell lung cancer: a randomized phase II trial. Ann Oncol 16:294-299

27. Wachters FM, Groen HJM, Biesma B et al (2005) A randomized phase II trial of docetaxel versus docetaxel and irinotecan in patients with stage IIIB-IV non-small-cell lung cancer who failed first-line treatment. Br J Cancer 17:15-20

28. Takeda K, Negoro S, Tamura T et al (2009) Phase III trail of docetaxel plus gemcitabine versus docetaxel in second-line treatment for non-small cell lung cancer: results of a Japan Clinical Oncology Group trial (JCOG0104). Ann Oncol 20:835-841

29. Scaliotti GV, Parikh P, von Pawel J et al (2008) Phase III study comparing cisplatin plus gemcitabine with cisplatin plus pemetrexed in chemotherapy-naïve patients with advanced stage nonsmall-cell lung cancer. J Clin Oncol 26:3543-3551

30. Lynch TJ, Bell DW, Sordella R et al (2004) Activating mutations in the epidermal growth factor receptor underlying responsiveness of non-small-cell lung cancer to gefitinib. N Eng J Med 350:2129 2139

31. Paez JG, Janne PA, Lee JC et al (2004) EGFR mutations in lung cancer: correlation with clinical response to gefitinib therapy. Science 304:1497-1500

32. Zang DY, Yang DH, Lee HW et al (2009) Phase I/II trial with docetaxel and S-1 for patients with advanced or recurrent gastric cancer with consideration to age. Cancer Chemother Pharmacol 63:509-516

33. Weiss GJ, Langer C, Rosell R et al (2006) Elderly patients benefit from second-line cytotoxic chemotherapy: a subset analysis of a randomized phase III trial of pemetrexed compared with docetaxel in patients with previously treated advanced non-small-cell lung cancer. J Clin Oncol 24:4405-4411 